\title{
The Joubert Syndrome Gene arl13b is Critical for Early Cerebellar Development in Zebrafish
}

\author{
Jian Zhu ${ }^{1,2,5} \cdot$ Han-Tsing Wang ${ }^{1,2} \cdot$ Yu-Rong Chen ${ }^{1,2} \cdot$ Ling-Ya Yan ${ }^{1,2}$ • \\ Ying-Ying Han ${ }^{1,2} \cdot$ Ling-Yan Liu ${ }^{1,2,3} \cdot$ Ying Cao ${ }^{4} \cdot$ Zhi-Zhi Liu $^{1,2,3}$. \\ Hong A. $\mathrm{Xu}^{1,2,3}$
}

Received: 16 October 2019/Accepted: 5 March 2020/Published online: 18 August 2020

(C) The Author(s) 2020

\begin{abstract}
Joubert syndrome is characterized by unique malformation of the cerebellar vermis. More than thirty Joubert syndrome genes have been identified, including $A R L 13 B$. However, its role in cerebellar development remains unexplored. We found that knockdown or knockout of arll $3 b$ impaired balance and locomotion in zebrafish larvae. Granule cells were selectively reduced in the corpus cerebelli, a structure homologous to the mammalian vermis. Purkinje cell progenitors were also selectively disturbed dorsomedially. The expression of atohl and ptfl, proneural genes of granule and Purkinje cells, respectively, were selectively down-regulated along the dorsal midline of the cerebellum. Moreover, wntl, which is transiently expressed early in cerebellar development, was selectively
\end{abstract}

Jian Zhu and Han-Tsing Wang have contributed equally to this work.

Electronic supplementary material The online version of this article (https://doi.org/10.1007/s12264-020-00554-y) contains supplementary material, which is available to authorized users.

Hong A. Xu

xuhong@ncu.edu.cn

1 Institute of Life Science, Nanchang University, Nanchang 330031, China

2 School of Life Sciences, Nanchang University, Nanchang 330031, China

3 Jiangxi Provincial Collaborative Innovation Center for Cardiovascular, Digestive and Neuropsychiatric Diseases, Nanchang 330031, China

4 Department of Molecular and Cell Biology, Tongji University School of Life Sciences and Technology, Shanghai 200092, China

5 Precise Genome Engineering Center, School of Life Sciences, Guangzhou University, Guangzhou 510006, China reduced. Intriguingly, activating Wnt signaling partially rescued the granule cell defects in arll $3 b$ mutants. These findings suggested that Arl13b is necessary for the early development of cerebellar granule and Purkinje cells. The arll3b-deficient zebrafish can serve as a model organism for studying Joubert syndrome.

Keywords Joubert syndrome $\cdot \operatorname{arl} 13 b \cdot$ Cerebellum . Development · Granule cell · Purkinje cell · Wnt

\section{Introduction}

Joubert syndrome (JS) is an autosomal-recessive neurodevelopmental disorder, which is characterized morphologically by the unique molar tooth sign, a complex malformation of the cerebellar vermis and brainstem, with abnormalities of axonal decussation affecting the corticospinal tract and superior cerebellar peduncles [1]. JS is clinically characterized by impaired motor functions and intellectual disability. Most cases of JS are variably associated with impairments of additional organs, including the retina, kidney, skeleton, and liver. More than 30 genes have been identified to cause JS (213300, Online Mendelian Inheritance in Man). Most of the proteins encoded by these genes are located in or near the primary cilium, an organelle found in eukaryotic cells, and this makes JS a typical ciliopathy [1]. Although malformation of the cerebellar vermis is common in JS, the role and mechanisms of the causative genes in cerebellar development have only been reported recently $[2,3]$.

Mutations of ARLI3B (HGNC ID 25419) lead to the classical form of JS [4]. The ARL13B gene encodes an enzyme belonging to the small GTPase superfamily and this makes it unique among the known causative genes of 
JS. It has been demonstrated that Arl13b is critical for processes of neural development, such as interneuron migration and placement [5], polarized radial glial scaffold formation [6], and neural tube patterning [7]. Arl13b might also be involved in photoreceptor degeneration and kidney cysts [8, 9]. However, the role of Arl13b in cerebellar development remains a mystery.

The zebrafish has been established as a model organism in studying JS [9, 10]. Here, we explored the role of $\operatorname{arll3b}$ in early development of the cerebellum and we hope use this powerful model to investigate the pathological mechanisms of JS and help to screen for therapeutic targets.

\section{Materials and Methods}

\section{Zebrafish Maintenance and Embryo Collection}

All zebrafish lines were raised and maintained under a photoperiod of $14 \mathrm{~h} / 10 \mathrm{~h}$ (light/dark) at $28.5^{\circ} \mathrm{C}$ in our facility supplied with filtered fresh circulating water. Wildtype zebrafish of the AB strain and the $a r l 13 b$ mutants were kindly provided by Dr. Ying Cao (Tongji University, Shanghai, China) and the $T g$ (neurodl:EGFP) transgenic zebrafish were a gift from Dr. Jing-Wei Xiong (Peking University, Beijing, China). arl13b homozygous mutants only survive up to 10 days, so heterozygous mutants were mated to produce homozygous embryos. The homozygous embryos were picked according to the curved tail since this phenotype is almost fully penetrant. The developmental stages of zebrafish were characterized following previously-described morphological criteria [11]. Fish embryos and larvae for in situ hybridization and immunostaining were raised in E3 supplemented with $0.003 \%$ phenylthiourea from $24 \mathrm{hpf}$ onward to prevent pigment formation. All handling procedures were approved by the Ethics Review Committee at Nanchang University.

\section{Morpholino Oligonucleotide Microinjection}

The morpholino (MO) antisense oligonucleotide blocking the translation of $a r l 13 b$ (5'-TTTCCCCCCTAAATGCTT TCACTGG-3') described previously [9] was purchased from Gene Tools LLC (Philomath, OR, USA). The MO was microinjected into zebrafish embryonic yolk at the one- to two-cell stage.

\section{Imaging of Zebrafish and Behavior}

The morphology of the zebrafish larvae (otolith and body curvature) was imaged at $4 \mathrm{dpf}$ using a Nikon AZ100 microscope (Nikon, Tokyo, Japan). The larvae were raised in Petri dishes and transferred to a new dish at $5 \mathrm{dpf}$ for behavioral analysis. After allowing adaptation to the new environment for $5 \mathrm{~min}$, locomotion was video-recorded for 3 min using a Nikon DS-Fill digital camera and processed with NIS-Elements F3.0 (Nikon).

\section{Whole-Mount In Situ Hybridization}

We made RNA probes from different templates: PCR products and linearized plasmid DNA. For PCR-based in situ templates, we designed PCR primers (listed in Supplemental Table 1) to amplify gene-specific products that contained the T3 promoter sequence, and RNA probes were transcribed in vitro using T3-RNA-polymerase. For the linearized plasmid DNA-based in situ templates, shh (HindIII/T7), atohla (Nco I /SP6), ptfla (Nco I/SP6), reelin (NcoI/SP6), and roraa (ApaI/SP6), the last four plasmids were kindly provided by Dr. Sheng-Ping L. Hwang (Institute of Cellular and Organismic Biology, Academia Sinica, Taipei, China). The antisense RNA probes were synthesized with T7 or SP6 RNA polymerase after plasmid DNA linearization. Whole-mount in situ hybridization was performed using digoxigenin-labeled antisense RNA probes and alkaline phosphatase-conjugated anti-digoxigenin antibodies (Roche, Mannheim, Germany), as described previously [12]. Embryos were mounted in glycerol, and images were captured using a Nikon AZ100 microscope.

\section{RNA Isolation and Quantitative Real-Time PCR (qPCR)}

Total RNA was extracted from the embryos using RNAiso Plus following the manufacturer's protocol (Takara, Shiga, Japan). Reverse-transcription reactions were carried out with M-MLV reverse transcriptase (Takara). qPCR assays were performed with SYBR Premix Ex Taq II (Takara) on the Abi-Step-One plus Real-Time PCR system (Applied Biosystems, Foster City, CA, USA). All the primer sequences used for qPCR are listed in Supplemental Table 2. All experiments were conducted at least three times. Student's $t$-test was applied to analyze the data and $P<0.05$ was indicated a statistically significant difference.

\section{Immunostaining}

For tubulin antibody staining, we fixed larvae with $2 \%$ trichloroacetic acid in PBS for $3 \mathrm{~h}$ at room temperature. For other antibodies, we fixed larvae overnight at $4{ }^{\circ} \mathrm{C}$ in $4 \%$ paraformaldehyde (PFA) in PBS supplemented with sucrose $(4 \% \mathrm{w} / \mathrm{v})$ [13]. The larvae were rinsed 3 times in $1 \times$ PBST $(1 \times$ PBS and $0.8 \%$ Triton $)$ for $10 \mathrm{~min}$ each. Afterward, larvae were dehydrated and rehydrated through graded methanols $(50 \% \mathrm{MeOH}$ once, $100 \%$ twice, and $50 \%$ 
once, $10 \mathrm{~min}$ each), and then rinsed 3 times in $1 \times$ PBST for 5 min each. The larvae were immersed in ice-cold acetone for $20 \mathrm{~min}$ at $-20^{\circ} \mathrm{C}$, rinsed 3 times in $1 \times$ PBST for $5 \mathrm{~min}$ each and digested with proteinase $\mathrm{K}$ for permeabilization. The concentration and treatment time of proteinase $\mathrm{K}$ was determined by the developmental stages of the larvae. After digestion, the larvae were re-fixed with fresh $4 \%$ PFA in $1 \times \mathrm{PBS}$ for $20 \mathrm{~min}$ at room temperature $(\mathrm{RT})$, and then rinsed 3 times in $1 \times$ PBST for $10 \mathrm{~min}$ each. The larvae were blocked with buffer containing $10 \%$ serum and $1 \%$ dimethylsulfoxide in $1 \times \mathrm{PBST}$ for $3 \mathrm{~h}$ at RT and incubated with primary antibody overnight at $4^{\circ} \mathrm{C}$. After rinsing 4 times in $1 \times$ PBST for $30 \mathrm{~min}$ each, the larvae were incubated with secondary antibody for $4 \mathrm{~h}$ at RT. The larvae were rinsed and counterstained with Hoechst 33342 (10 $\mu \mathrm{g} / \mathrm{mL}$ ) for nuclear staining. The following primary antibodies were used: mouse anti-tubulin (1:1000, T6793, Sigma), mouse anti-parvalbumin (1:1000, MAB1572, Chemicon), goat 3A10 (1:1000, DSHB), and goat antiGFP (1:500, 600-101-215, Rockland). For fluorescence detection, we used Alexa Fluor 488 donkey anti-goat IgG $(\mathrm{H}+\mathrm{L})(1: 500, \mathrm{~A} 11055$, Invitrogen) and Alexa Fluor 488 donkey anti-mouse IgG $(\mathrm{H}+\mathrm{L})$ (1:500, A21202, Invitrogen). Fluorescence images of larvae were acquired using an Olympus FV1000 confocal microscope, and Z-series stacks were shown as two-dimensional projections.

\section{Lithium Treatment}

Embryos at 30-37 hpf were incubated in E3 containing $\mathrm{LiCl}$ (A100416-0025, Sangon) at a final concentration of $50 \mathrm{mmol} / \mathrm{L}$. Age-matched untreated embryos served as controls.

\section{Results}

\section{Depletion of arl13b Impairs Posture and Locomotion in Zebrafish Larvae}

In order to explore the function of ARL13B in vivo, we started to investigate whether the neurological features of JS are mimicked in arl $13 b$ mutant zebrafish, an established model organism [4, 9]. The null mutation of $a r l 13 b$ $\left(\operatorname{arl} 13 b^{-/-}\right)$, identified in a retroviral insertion screen, led to body axis curvature (Fig. S1) [4, 14]. The curved tail phenotype is almost fully penetrant in homozygous embryos while it is rarely found in wild-type and heterozygous embryos [4]. We took advantage of this readilyrecognizable morphological phenotype to pick homozygous mutants for further experiments. The picked embryos were genotyped by PCR which confirmed retroviral insertion into the first exon of the $a r l 13 b$ gene.
Furthermore, qPCR results demonstrated that the expression of $a r l 13 b$ mRNA was almost undetectable in the picked embryos, while it was normally-expressed in wildtype embryos (Fig. S3). The arl13b ${ }^{-/-}$mutant larvae [5 days post-fertilization (dpf)] were usually motionless and occasionally moved by trembling and circling. These abnormal movements were not found in wild-type larvae, which swam around freely and elegantly (Movie 1).

To assess the phenotype specificity and confirm the locomotor defects, we performed transient knockdown experiments with an antisense morpholino oligonucleotide (MO) designed to specifically block the translation of Arl13b [9]. After injecting $9.7 \mathrm{ng}$ arl13b MO into embryos, we observed body curvature and abnormal locomotion like that in $\operatorname{arll}_{13 b^{-/}}$mutants (Movie 1). Considering that the curvature might interfere with locomotion, we injected a subthreshold dose of MO (7.3 ng) and found that the larvae showed no apparent body axis defects while they still exhibited impairments in posture and locomotion (Movie 1). Wild-type larvae maintained a normal posture (Fig. 1A). In contrast, the subthreshold-dose morphants usually laid on their sides, i.e., lost posture (Fig. 1B, C). As for the swimming patterns, wild-type larvae often exhibited spontaneous swimming characterized by small bending angles (Fig. 1D). However, the subthreshold-dose morphants swam with trembling and exhibited large bending angles (Fig. 1E). The subthreshold-dose results suggested that the posture and locomotion defects in arl13b mutants and morphants are due to the depletion of $a r l 13 b$ rather than body curvature.

In addition, the sensation of arl13b mutants and morphants was compromised; they were not sensitive to a needle poke, while wild-type embryos were sensitive and swam away quickly (Movie 1).

Taken together, the above results suggested that zebrafish deficient in arl $13 \mathrm{~b}$ exhibit impairments in posture and swimming pattern, reminiscent of the signs of JS.

\section{Depletion of arl13b Results in Morphological Defects of the Cerebellum}

The above behavioral defects suggested the arll3b-deficient zebrafish might serve as a good model for studying JS. We first investigated the development of the cerebellum since it is the main cause of the characteristic molar tooth sign in JS. The outline of the cerebellum can easily be distinguished by immunostaining with an anti-tubulin antibody (Fig. 1F). Obvious morphological defects were found in the cerebella of both $a r l 13 b$ mutants and morphants, and the antibody-labeled fibers were globally reduced (Fig. 1G, H). The posterior outline of the cerebellum was thinner than that of the wild-type and invaginated anteriorly at the midline, while the anterior outline was 

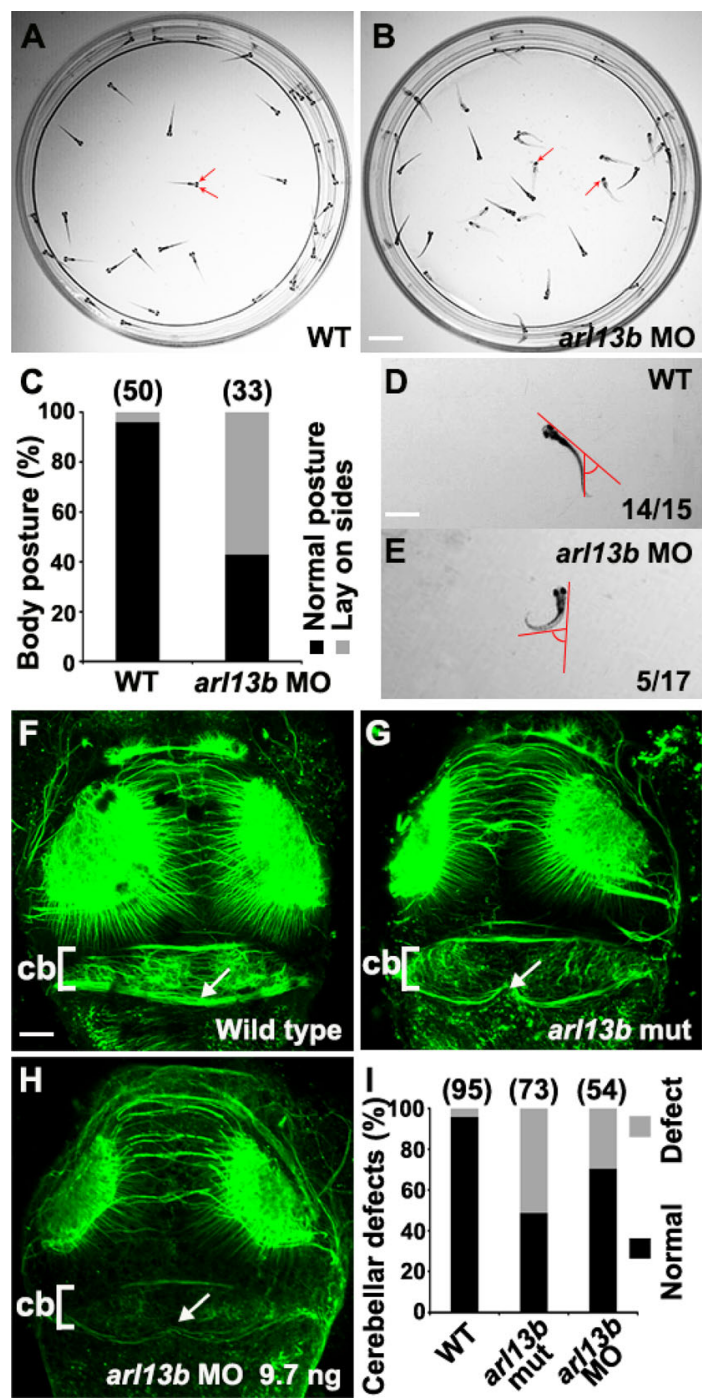

Fig. 1 Knockdown of arll3b impairs posture, locomotion, and cerebellar morphology in zebrafish larvae. A Wild-type sibling larvae remain vertically oriented at 5 days post-fertilization (dpf), with both eyes visible from a top view (arrows). B In contrast, larvae injected with $\operatorname{arl} 13 b \mathrm{MO}$ (subthreshold dose, $7.3 \mathrm{ng}$ ) often lie on their side at the bottom of the dish, with only one eye visible (arrows). Note that the body of the subthreshold-dose arl13b morphants are relatively straight and only slightly curved. C Statistics of the posture of zebrafish larvae at $5 \mathrm{dpf}$. D Wild-type larvae perform stereotyped spontaneous swimming with small bending angles. E The arl13b morphants (subthreshold dose, $7.3 \mathrm{ng}$ ) swim slower and exhibit greater bending angles. F-H Immunostaining with acetylated tubulin antibody outlines the cerebellum of larvae at $3 \mathrm{dpf}$. Comparing the dorsal view of wild-type embryos $(\mathbf{F})$ with $\operatorname{arll} 3 b$ mutants $(\mathbf{G})$ reveals morphological defects of the cerebellum (arrows). The cerebellar defects were also present in embryos injected with arll $3 b \mathrm{MO}$ (H) (cb, cerebellum). I Statistics of the embryos with morphological defects of the cerebellum. The number of embryos examined in each condition is indicated above each column. A-E, Scale bar $1 \mathrm{~cm} . \mathbf{F}-\mathbf{H}$, Scale bar $100 \mu \mathrm{m}$.

relatively normal (Fig. 1F-I). Moreover, the acetyl-tubulin-positive parallel fibers connecting the cerebellar hemispheres were disturbed in arll3b-deficient larvae while the commissural axons between the optic tecta remained largely unaffected (Fig. 1F-H). The midline cerebellar defects in $\operatorname{arl} 13 \mathrm{~b}$ mutants and morphants are reminiscent of the midline cerebellar defects in JS patients [2].

Besides the cerebellum, we also checked other neural tissues. $\operatorname{arll} 13 b$ is highly expressed in the ventricle and otic vesicle at early developmental stages [9]; the ciliated cells in the inner ear are important for the formation of otoliths [15]. Usually, there are two otoliths in zebrafish (Fig. S1). We found that the otoliths displayed defects in number and size in both arll3b mutants and morphants (Fig. S1). The otoliths are critical for proper balance and hearing, and their impairment might contribute to the postural and locomotor defects in arl13b-depleted larvae. We also checked motor axons and Mauthner axons, which are involved in locomotion. No morphological defect was observed in either acetylated tubulin-positive motor axons or 3A10-positive Mauthner axons (Fig. S2). We focused on the cerebellum in the subsequent experiments since cerebellar malformation is a major hallmark of JS.

\section{Depletion of arl13b Impairs Granule Cell Progeni- tors in the Developing Cerebellum}

The morphological defects in arll3b-deficient embryos prompted us to further investigate the role of $\operatorname{arll} 13 \mathrm{~b}$ in the development of cerebellar neural circuits. Like mammals, the zebrafish cerebellum is derived from the dorsal part of the anterior hindbrain [16]. The cerebellum is composed of several types of neurons, which are categorized according to their major neurotransmitter, glutamate or GABA. The glutamatergic granule neurons are derived from granule cell progenitors located in the upper rhombic lip (URL). Granule cells are the most abundant type of neuron in the cerebellum. We first examined the expression of the granule progenitor cell marker atohl using whole-mount in situ hybridization. In zebrafish, there are three paralogues of atohl - $1 a, 1 b$, and $1 c$ - which are expressed sequentially in overlapping but distinct granule cell progenitors within the rhombic lip [17, 18]. In wild-type embryos, atohla was strongly expressed in the URL and the lateral rhombic lip (Fig. 2A). In some arll3b morphants, atohla was absent from the anterior dorsomedial URL along the midline (Fig. 2C). The specific absence of atohla was maintained at 48 hours post-fertilization (hpf) (Fig. 2A'-C'), suggesting that the phenotype could not be due to developmental delay. No detectable change of atohlb was found (Fig. 2D-F'), indicating that the absence of atohla dorsomedially was specific and not due to defects in cerebellar structure. The expression level of atohlc was decreased in arl $13 b$ morphants at $2.5 \mathrm{dpf}$ and 4 dpf (Fig. 2G-I'). In arll3b mutants, atohlc was decreased slightly at $2.5 \mathrm{dpf}$ (Fig. 2H). The expression defects of 
Fig. 2 The expression of markers of cerebellar granule cell progenitors is impaired in arl13b-deficient embryos. A-I' Representative images of in situ hybridization illustrate that the three paralogues of atohl (ato$h l a, l b$ and $l c)$ are expressed in distinct populations of cerebellar granule cell progenitors. atohla is expressed in the URL and LRL. Similar expression patterns of atohla occur in wild-type embryos (A) and ar$l 13 b$ mutants $(\mathbf{B})$ while its expression is absent from the oral dorsomedial URL (dashed box) in arl13b morphants (C) at $36 \mathrm{hpf}$ and $48 \mathrm{hpf}\left(\mathbf{A}^{\prime}-\mathbf{C}^{\prime}\right)$. The expression patterns of atoh $1 b$ remained unaffected in $\operatorname{arl} 13 b$ mutants and morphants (D-F'). The expression level of atohlc was decreased in the URL in arl13b morphants (I and I') (cb, cerebellum; URL, upper rhombic lip; LRL, lower rhombic lip). A-I', Scale bar $100 \mu \mathrm{m}$.

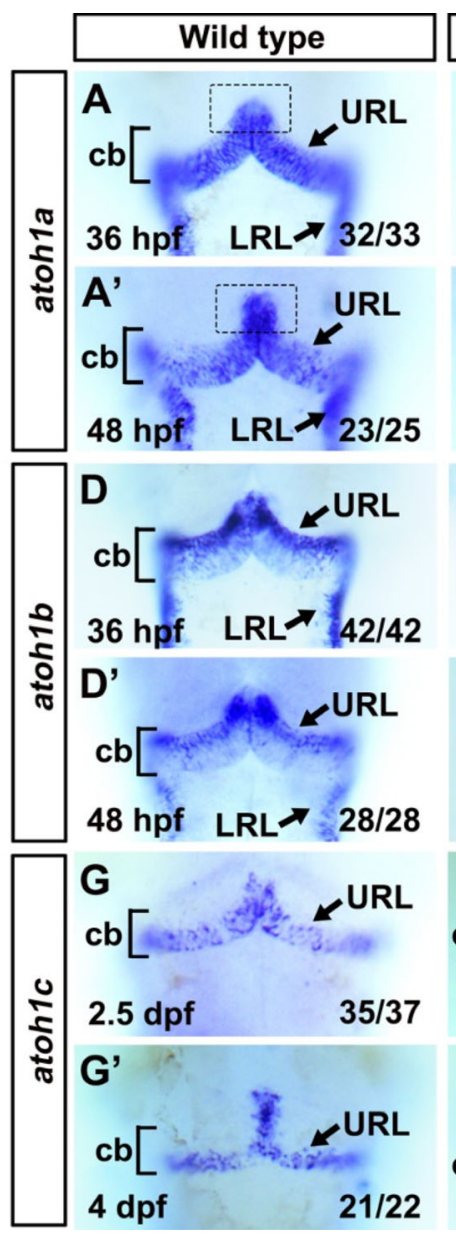

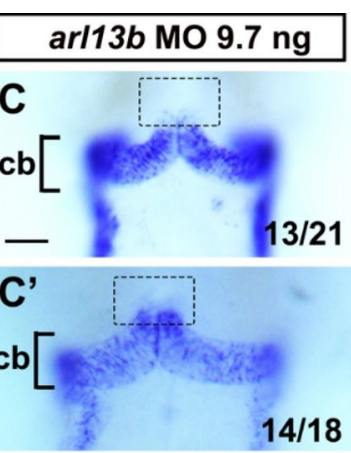
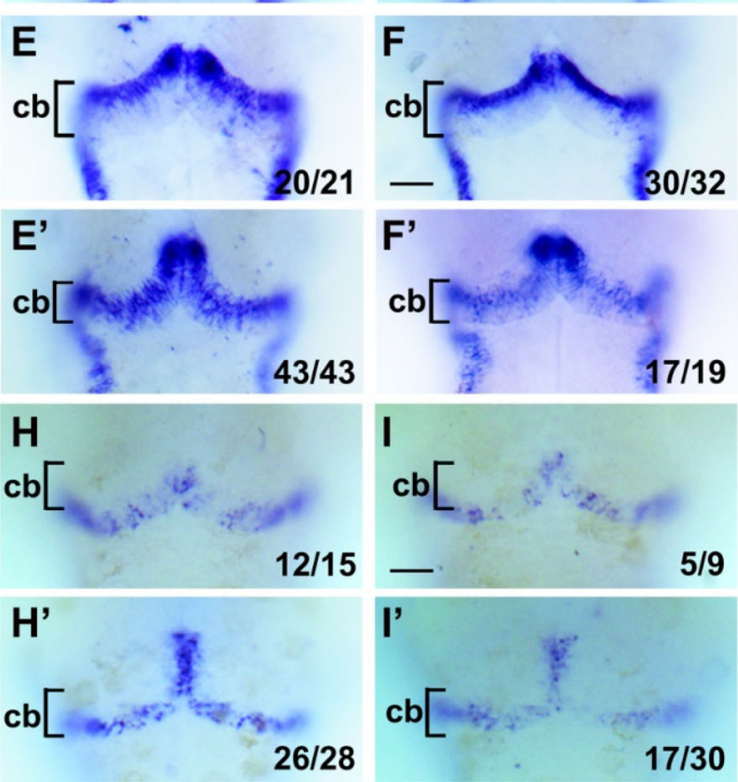

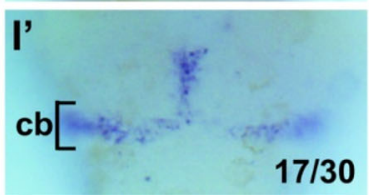

atohla and atohlc were more apparent in arll3b morphants than in mutants. This could be due to the maternally-deposited arll $3 b$ mRNA in null mutants which might mask the early defects caused by Arl13b deficiency [9]. The MO blocks the translation of arll3b mRNA, including the maternally-derived mRNA, so the phenotypes in morphants are more penetrant than in mutants [9]. However, potential off-target effects of the MO could not be excluded although it seems unlikely since most phenotypes have been reported in arll3b mutants [9] (and our data).

We performed quantitative PCR (qPCR) using whole body tissues to verify the above results. atohlc was consistently down-regulated in arll $3 b$ mutants from $36 \mathrm{hpf}$ to $4 \mathrm{dpf}$ (Fig. S3). However, atohla and $1 b$ were upregulated at $36 \mathrm{hpf}$ but down-regulated at later stages, when comparing arl13b mutants with the wild-type (Fig. S3). It should be noted that we used the whole zebrafish body and the qPCR results represented the mixed expression of all tissues. According to our in situ hybridization results and those of others [17, 18], atohlc is mainly expressed in the URL (Fig. 2). The decrease of atohlc in arl13b mutants revealed by qPCR indicated that atohlc is reduced mainly in the URL and confirmed our in situ hybridization results.

atohlc and $1 a$ are critical for the full complement of granule cells in the corpus cerebelli (CCe), a structure homologous to the mammalian cerebellar vermis $[17,19]$. The decrease of atohla and particularly atohlc revealed by in situ hybridization and qPCR in arl13b-deficient embryos might interfere with the development of granule cells in the CCe.

Zic1 has been shown to be involved in mouse granule cell proliferation [20]. We found that $z i c l$ was expressed in the URL cells in wild-type zebrafish at $48 \mathrm{hpf}$ while it was dramatically down-regulated in arl $13 b$ morphants and mutants (Fig. 3A-C).

These results suggested that Arl13b participates in cerebellar development by regulating the development of granule cell progenitors. 

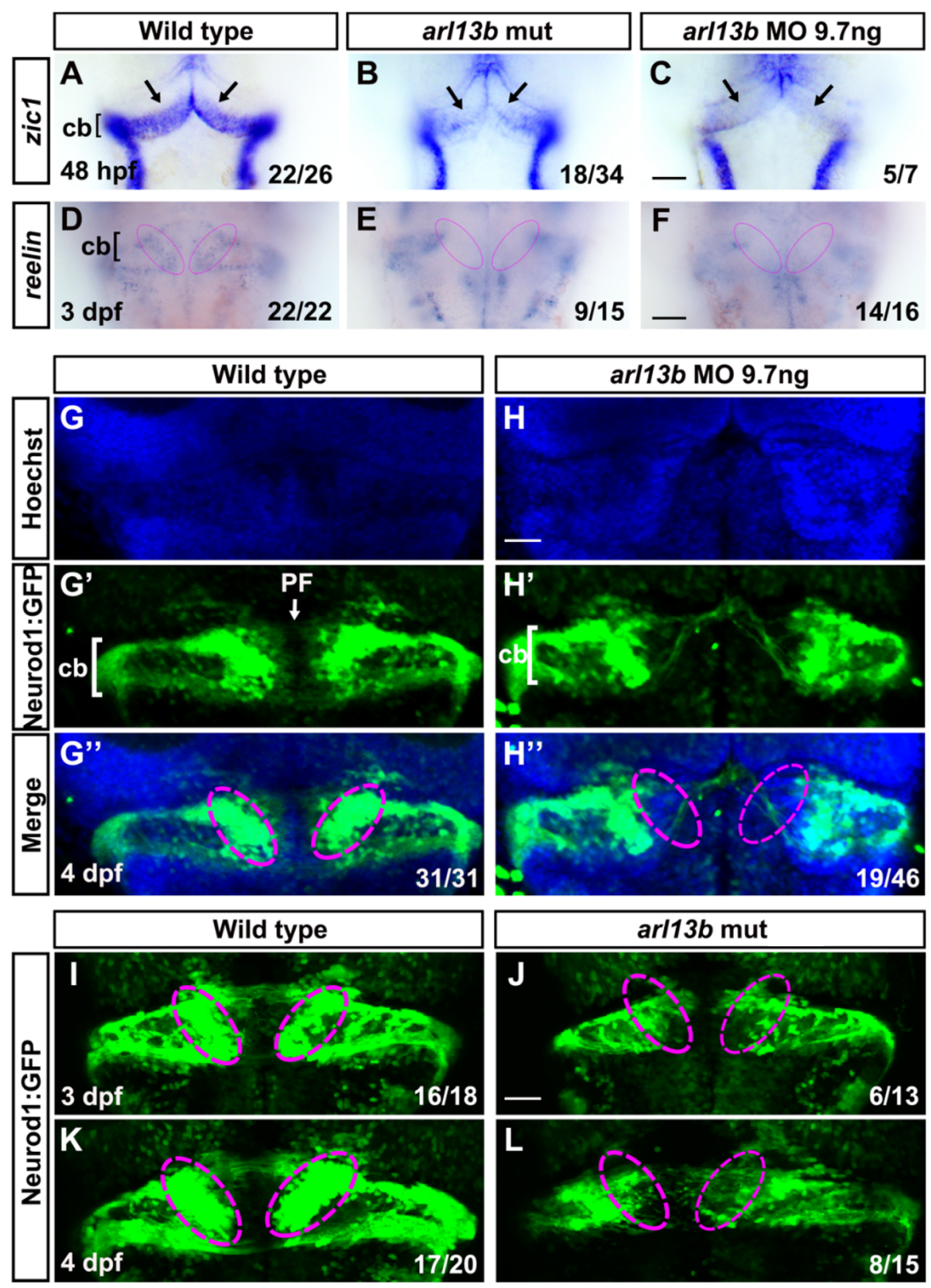

Fig. 3 Disruption of arl13b impairs the development of cerebellar granule cells. A Expression of the granule cell progenitor marker, zicl, in the cerebellum of wild-type embryos at $48 \mathrm{hpf}$ revealed by in situ hybridization. $\mathbf{B}, \mathbf{C}$ Expression of zicl is reduced in the URL of both arl13b mutant and morphant embryos compared with wild-type embryos. Note that zicl expression is severely reduced in the dorsomedial subregions of the URL (arrows). D The expression of the differentiated granule cell marker, reelin, in the cerebellum of wildtype embryos at $3 \mathrm{dpf}$. E, F Expression of reelin is reduced in the cerebellum of both arl $13 \mathrm{~b}$ mutant and morphant embryos. Note that in some embryos reelin expression is almost absent in the dorsal medial subregions of the cerebellum (colored ovals). G-G' In
Tg(neurod1:eGFP) transgenic embryos, GFP+ granule cells are grouped into three clusters, the dorsomedial (dashed ovals), dorsoposterior, and ventrolateral subdivisions. H-H" The pattern of GFP+ granule cells is dramatically altered in the cerebellum, and particularly in the dorsomedial cerebellar subdivisions (dashed ovals) are severely affected in arl13b morphants. The parallel fibers connecting the two hemispheres are disrupted in $a r l 13 b$ morphants. Dorsal views of the embryos are shown. I-L Malformations of the dorsomedial cerebellar subdivisions (dashed ovals) and parallel fibers are also present in $\operatorname{arl} 13 \mathrm{~b}$ mutants both at $3 \mathrm{dpf}$ and $4 \mathrm{dpf}$. cb, cerebellum; PF, parallel fiber. A-F, Scale bar $100 \mu \mathrm{m}$. G-L, Scale bar $50 \mu \mathrm{m}$. 


\section{Depletion of $a r l 13 b$ Impairs Granule Neurons Specifically in the Corpus Cerebelli}

The impairment of granule cell progenitors might interfere with the subsequent development of granule neurons, so we next examined the differentiation of granule neurons. We found that reelin, a marker of differentiated granule cells [21], was markedly decreased in the cerebellum of arl13bdeficient embryos, particularly in the dorsomedial subregions (Fig. 3D-F). It has been demonstrated that $N E U$ ROD1 is expressed in immature and mature cerebellar granule neurons in both mammals and zebrafish [18, 22]. We found that neurodl was absent from the dorsomedial cerebella of arl13b morphants (Fig. S4) while the dorsolateral neurons were not affected, similar to the expression of reelin. We further examined the development of cerebellar granule cells using the transgenic line $\mathrm{Tg}(\mathrm{neu}$ rod1:EGFP) [23]. In these embryos, GFP was expressed in three main clusters: two close to the midline, the dorsomedial and dorsoposterior granule cells, and one distant from the midline, the ventrolateral granule cells (Fig. 3GG'). The patterns of the Tg(neurodl:EGFP) GFP+ granule cells resembled the granule cells labeled by $T g$ (gatal:GFP) [19]. Knocking down arl13b in $T g$ (neurodl:EGFP) embryos caused a global reduction of GFP+ granule neurons compared to the control transgenic embryos. Particularly, the dorsomedial clusters were severely affected and, in some embryos, these clusters were totally absent (Fig. 3H-H'). The ventrolateral and dorsoposterior clusters were still present though with reduced numbers of GFP+ neurons (Fig. 3H-H'). These results were consistent with the expression pattern of neurodl detected by in situ hybridization (Fig. S4). The dorsomedial subdivision of granule neurons populate the CCe $[17,19]$. The defects of CCe granule cells in arll $3 b$ deficient zebrafish resembled the defects in the cerebellar vermis found in JS patients. Tg(neurodl:EGFP) labeled some parallel fibers at the midline of cerebellum (Fig. 3G'). Upon arll3b knockdown, the parallel fibers were dramatically disrupted (Fig. 3H'), similar to those found by anti-tubulin immunostaining (Fig. 1F-I). These phenotypes were also found in arll3b mutants (Fig. 3I-L). The arl13b mutants were crossed with $T$ g(neurodl:EGFP) fish and transgenic labeled arll $3 b$ homozygotes were picked. The selective malformation of CCe granule cells and disruption of parallel fibers were frequently observed in arll3b mutants both at $3 \mathrm{dpf}$ (Fig. 3I, J) and $4 \mathrm{dpf}$ (Fig. 3K, L).

The qPCR results revealed that the markers of differentiated granule cells calbindin $2 a$ (calretinin, calb2a) and GABA receptor alpha 6a (gabra6a) were dramatically reduced in $\operatorname{arll} 3 b$ mutants (Fig. S3). These results suggested that not only the proliferation and migration but also the differentiation of granule cells is impaired in $a r l l 3 b$ deficient embryos.

\section{Depletion of $a r l 13 b$ Impairs the Development of Cerebellar Purkinje Cells}

Both the function and development of the cerebellum are dependent on the cooperation between granule neurons and Purkinje cells, two major neuronal types in the cerebellum, so we further investigated the role of $a r l 13 b$ in the development of Purkinje cells. ptfla was used as a marker to label the precursors of Purkinje cells by in situ hybridization and it was found to be expressed in the ventricular zone of wild-type embryos at $48 \mathrm{hpf}$ (Fig. 4A). In $a r l 13 b$ mutant and morphant embryos, the expression level of ptfla was dramatically and selectively reduced in the dorsomedial ventricular zone, while it was relatively normal in the ventrolateral regions (Fig. 4B, C). The phenotype of morphants was more penetrating than that of mutants, as found in granule cells. These results demonstrated that the progenitors of Purkinje cells are selectively reduced dorsomedially in arl $13 b$-deficient embryos. We further examined differentiating Purkinje cells using roraa as a marker [24]. This revealed that the dorsomedial clusters of differentiating Purkinje cells were dramatically reduced, while the ventrolateral clusters were only mildly reduced in arll3b-deficient embryos at $3 \mathrm{dpf}$ (Fig. 4D-F). This is reminiscent of the selective reduction of dorsomedial granule cell clusters (Fig. 3). A similar decrease of dorsomedial clusters was observed at $4 \mathrm{dpf}$ (Fig. 4G-I). As Purkinje cells differentiate and mature, early distinct clusters of Purkinje cells merge together, become unified, and form a continuous layer spanning the mediolateral width of the cerebellum [25]. Immunostaining with an antibody against parvalbumin was used to label differentiated Purkinje cells and demonstrated that they were dramatically reduced globally in the cerebellum at $5 \mathrm{dpf}$, including both the dorsomedial and ventrolateral clusters (Fig. 4K-L'). qPCR results revealed that the markers of immature and mature Purkinje cells roraa, aldoca, and pvalb7 were reduced (Fig. S3D), consistent with the immunostaining results.

The global reduction of mature Purkinje cells differed from the specific reduction of dorsomedial clusters of granule cells at the late larval stage. The early specific dorsomedial defects of both Purkinje progenitors and granule progenitors are likely due to unknown common defects in the mechanisms required and partly shared by the two types of neural progenitor. 
Fig. 4 Disruption of arl13b reduces both precursor and differentiated cerebellar Purkinje cells. A-I Representative images of in situ hybridization using anti-sense probes against ptfla and roraa to label Purkinje precursors and differentiated cells, respectively. The dorsomedial clusters of precursor of Purkinje cells and differentiated Purkinje neurons are selectively reduced (arrows). $\mathbf{J}-$ L' Immunostaining of mature Purkinje neurons using antiparvalbumin antibody reveals that the dorsomedial Purkinje neurons are reduced in arll3bdeficient embryos. A-I, Scale bar $100 \mu \mathrm{m}$. J-L"' Scale bar 50 $\mu \mathrm{m}$.

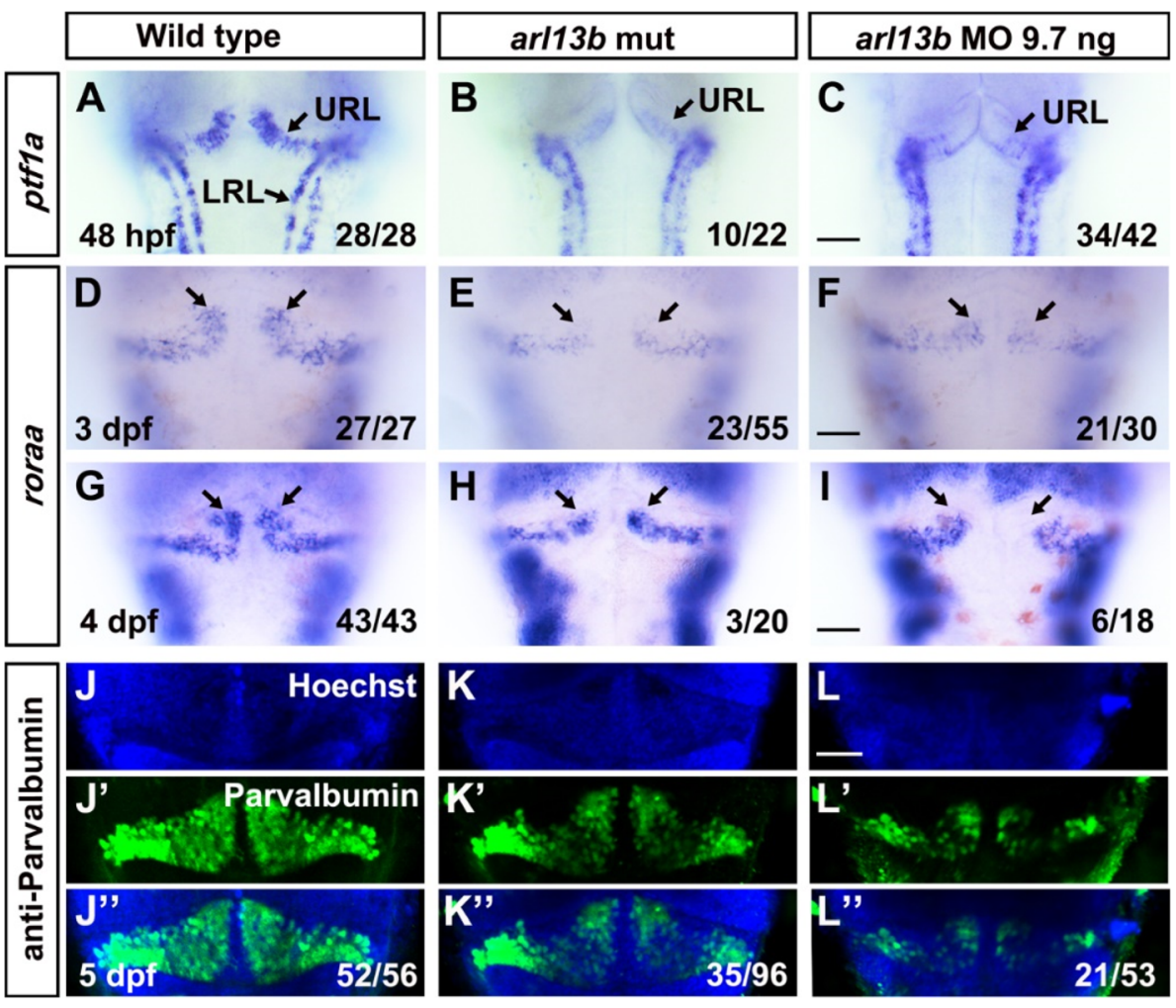

\section{Depletion of arl13b Reduces wnt1 Expression in the Developing Cerebellum}

Arl13b has been demonstrated to be associated with Hedgehog signaling pathways [7, 26, 27], so we examined the expression of Hedgehog signaling components by in situ hybridization and qPCR. shh was not detected in the cerebellar anlage at $24 \mathrm{hpf}$ although it was ventrally expressed along the neural tube (Fig. S5). No detectable change of $s h$ expression in the cerebellum was observed in $\operatorname{arll} 3 \mathrm{~b}$ morphants, although its expression in the zona limitans intrathalamica was reduced dorsally (Fig. S5). The expression level of the hedgehog receptor patchedl was relatively low (Fig. S5). It was hard to tell whether it was expressed in the cerebellum in wild-type embryos at $30 \mathrm{hpf}$ as well as whether its expression level or pattern changed. We then used qPCR to assess the expression levels of Shh signaling components. The expression of the receptors smoothen (smo) and patchedl (ptchl), and the Hedgehog signaling components gli2a, $g l i 2 b$, and gli3 were dynamically regulated in the early stages of cerebellar development (Fig. S5). These results suggested that Hedgehog signaling components are altered globally in arl $3 b$ mutants while no detectable change occurs early in cerebellar development.

Wnt signaling is also critical for cerebellar development [28] and cooperates with Hedgehog signaling to regulate cerebellar olig2+ cell development [29]. Intriguingly, recessive mutations of human $W N T 1$ result in hypoplasia of the cerebellum, particularly of the vermis, suggesting a conserved role of WNT1 in vermis development [30]. Besides being mainly expressed at the midbrain-hindbrain boundary, in mice wntl is also transiently expressed in the URL [31], from which many cerebellar neurons are derived. The transient expression of wntl in the URL is conserved in zebrafish [29]. We found that wntl was transiently expressed in the dorsal cerebellum at $30 \mathrm{hpf}$ in wild-type embryos (Fig. 5A). Its expression was strikingly reduced and almost absent from the cerebellum of some arll3b mutants (Fig. 5B), indicating that Wnt signaling is disrupted early in cerebellar development.

\section{Activating Wnt Signaling Partially Rescues Cere- bellar Defects in arl13b Mutants}

We started to explore whether activating Wnt signaling with lithium, an agonist of Wnt signaling, can mitigate the cerebellar defects in arl13b-deficient embryos. The dose of lithium chloride $(\mathrm{LiCl})$ was optimized for different developmental stages. We found that treating the embryos with $50 \mathrm{mmol} / \mathrm{L} \mathrm{LiCl}$ at $30-37 \mathrm{hpf}$ reduced the cerebellar structural defects in arll3b mutants. The wild-type embryos treated with $\mathrm{LiCl}$ at the same time showed no detectable cerebellar defects (Fig. 6A-E). We further examined the rescue effects of $\mathrm{LiCl}$ with $\mathrm{Tg}$ (neurodl:EGFP) embryos and found that the dorsomedial 


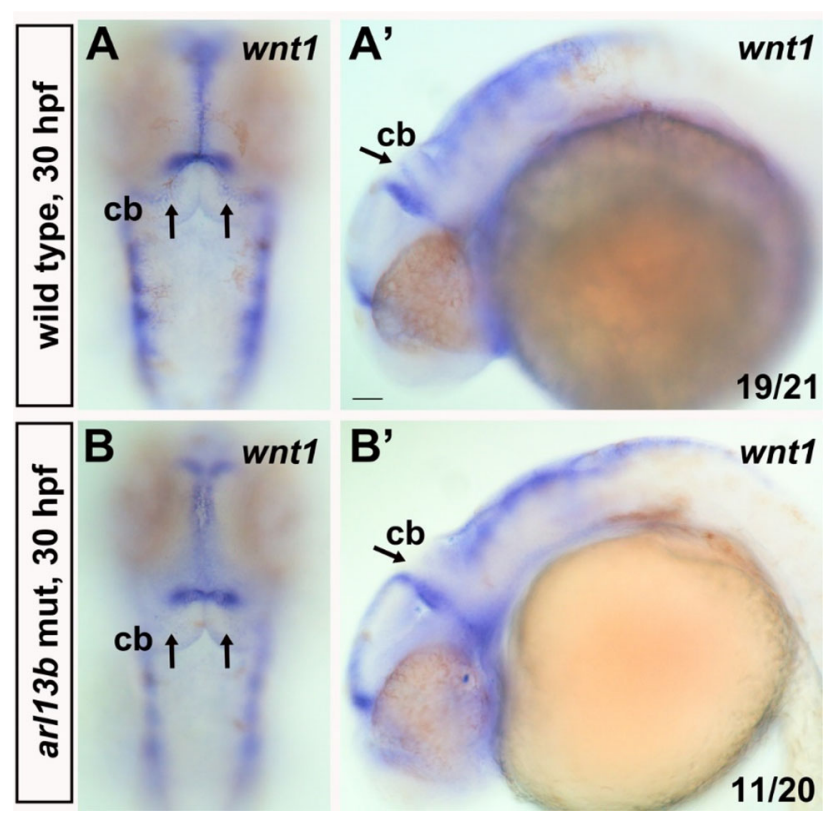

Fig. 5 Wnt1 is selectively down-regulated in the cerebellum of arl $13 \mathrm{~b}$ mutants. wnt 1 is transiently expressed in the cerebellum of WT embryos (A, $\left.\mathbf{A}^{\prime}\right)$ (arrows) while it is dramatically reduced in $\operatorname{arll} 13 b$ mutants (B, B'). A and B, dorsal view; A' and B', lateral view. Note that the expression of wntl is selectively decreased in the cerebellum while its expression at the midbrain-hindbrain boundary is intact. AB', Scale bar $100 \mu \mathrm{m}$.

reduction of granule cells caused by arll $3 b$ knockdown was mitigated by $\mathrm{LiCl}$ treatment (Fig. 6F-J).

\section{Discussion}

Most published papers study the role of JS genes during late embryonic or postnatal development of the cerebellum, though the molar tooth sign can be detected early in the first trimester [32]. Moreover, these studies mainly focus on cerebellar granule cells. In this study, we found that the disruption of arll $3 b$ in zebrafish larvae leads to early cerebellar malformations and defects in both granule and Purkinje cell progenitors. The early developmental patterning of cerebellar granule neurons is compromised particularly in the dorsomedial subregions of the $\mathrm{CCe}$, a structure homologous to the mammalian cerebellar vermis. This phenotype is reminiscent of hypoplasia of the cerebellar vermis in JS. Molecular and cellular studies revealed that the early development of the progenitors of both granule cells and Purkinje cells are selectively altered in arll3b-deficient embryos. Arl13b may be involved in regulating a network of signaling pathways, including Wnt and Atoh1. Treating the arl $13 b$ mutants with $\mathrm{Li}^{+}$, an agonist of Wnt signaling, partially rescued the cerebellar defects.

Our finding that granule neurons are reduced specifically in the dorsomedial subregions of the $\mathrm{CCe}$ in arll3bdeficient zebrafish larvae is consistent with the enrichment of $\operatorname{arll} 3 \mathrm{~b}$ expression in the ventricle at early stages, from $25 \mathrm{hpf}$ to $40 \mathrm{hpf}$ and later [9,33]. This finding is also consistent with reports that granule neurons are reduced
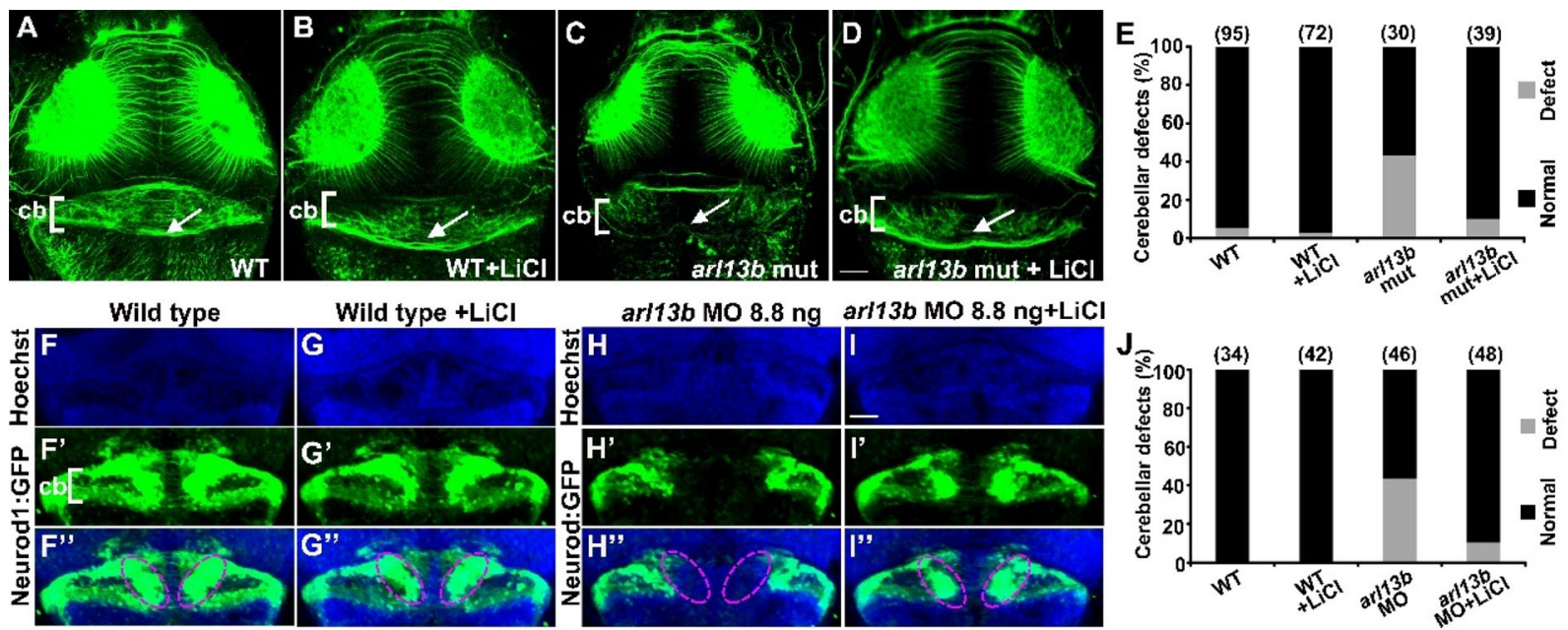

Fig. 6 Treating the arl13b mutants with lithium mitigates the morphological defects in the cerebellum. A-D Representative images of embryos treated with $50 \mathrm{mmol} / \mathrm{L} \mathrm{LiCl}$ at $30-37 \mathrm{hpf}$, fixed at $3 \mathrm{dpf}$, and immunostained with anti-tubulin antibody to reveal cerebellar morphology. Treatment of wild-type embryos with $\mathrm{Li}^{+}$does not affect the cerebellar morphology (A, B) (arrows). The morphological defects of the cerebellum in arl13b mutants treated with $\mathrm{Li}^{+}$are partially rescued (C, D) (arrow). A-D, Scale bar $100 \mu \mathrm{m}$. E Statistics revealing that the proportion of arl13b mutant embryos with cerebellar defects is dramatically decreased by $\mathrm{LiCl}$ treatment. $\mathbf{F}-$ I' Representative images of $\mathrm{Tg}$ (neurod1:EGFP) transgenic embryos used to label granule cells. Treating wild-type transgenic embryos with $\mathrm{Li}^{+}$causes no defect (F-G') (dashed ovals). Treating arl13b morphant transgenic embryos restores the dorsomedial cluster of granule cells (H-I') (dashed ovals). F-I', Scale bar $100 \mu \mathrm{m}$. J The proportion of $\operatorname{arll} 3 \mathrm{~b}$ morphant embryos with cerebellar defects in the dorsomedial clusters is dramatically reduced by $\mathrm{LiCl}$ treatment. 
along the anteroposterior but not the mediolateral axis in mouse mutants of the cilia genes Kif3a, Ift8, or Rpgripll $[34,35]$. The reduction of granule neurons in these mutants could be caused by proliferation defects in granule progenitors [34, 35]. Although the cerebellar granule neurons in Ahil- or Cep290- knockout mice are only slightly affected, there are significant proliferation defects of granule cells at an early developmental stage (E16.5)[2]. Proliferation defects of granule progenitors seem to be common in these cilia gene mutants [3, 34, 35]. The reduction of granule neurons in arll3b-deficient zebrafish was likely caused by proliferation defects in granule progenitors since the expression pattern of atohla was altered and atohlc was dramatically down-regulated (Fig. 2 and S3). Atoh1 is known to be critical for cerebellar granule neurogenesis in the mouse [36] and for the proliferation of granule cells in zebrafish [18]. atohlcderived cells contribute to the majority of granule neurons in the $\mathrm{CCe}$, while a minority is derived from atohla progenitors [17, 18]. The dramatic down-regulation of atohlc and the selective hypoplasia of the CCe in arll3bdeficient embryos are consistent with the critical role of atohlc in populating granule neurons in the CCe [17]. The dramatically decreased expression of zicl and neurodl specifically in the dorsomedial subregions of the cerebellum (Figs. 2 and S4) is also consistent with the proliferation defects of granule progenitors since both genes are implicated in granule cell proliferation [18, 20]. We immunostained the embryos using anti-pH3 antibody, a marker of cell proliferation, and found reduced fluorescence intensity of pH3-positive cells in the cerebellum of arl13b mutants (Fig. S6). These results are consistent with potential proliferation defects of granule cells in the cerebellum.

It has been shown that Arl13b regulates the migration and location of interneurons [5]. The specific hypoplasia of the dorsomedial subregions of the $\mathrm{CCe}$ in arl13b-deficient zebrafish could also be due to migration defects in granule cell precursors. It has been demonstrated that the URL generates different populations of granule cell precursors along its mediolateral axis. These precursors migrate along different routes and form different functional compartments of the mature cerebellum: the eminentia granularis and the CCe [19]. Atoh1 has been shown to be critical for the migration of granule cell precursors out of the URL in mice and particularly Atoh1c in zebrafish [17, 36]. The reduction of atohlc expression in arl13b-deficient zebrafish could lead to migration defects of the granule cell precursors. The migration defects might impair the differentiation of granule neurons since several markers of granule neurons are altered, particularly neurod1. The absence of $T g$ (neurodl:EGFP) + cells in the dorsomedial domain of the cerebellum in arl13b mutants differs from that seen in atohlc mutants [17], in which neurodl is expressed in most of the $\operatorname{Tg}($ atohlc:kaede $)+$ granule cell progenitors, including the dorsomedial cells along the midline. These results suggest that depletion of $a r l 13 b$ also disrupts other signaling pathways besides Atoh1.

Wnt1 is transiently expressed in the cerebellum of zebrafish [29] (Fig. 5) and mouse [31] and its mutations in humans result in hypoplasia of the cerebellar vermis [30], indicating that Wnt signaling is a potential mechanism underlying the pathology of arl13b mutant zebrafish and humans. Besides wntl, other wnt genes have also been detected in the cerebellum, such as wnt3, wnt7a and wnt10b [37], which might be involved in early cerebellar development [3, 38, 39]. Further study is required to investigate whether they are also regulated by Arl13b. The Wnt downstream signaling components $\beta$-catenin and Gsk $3 \beta$ regulate the transcription and protein stability of Atoh1 [40, 41]. Wnt1 has been proposed to regulate Atoh1 expression in the developing cerebellum [31]. These reports are consistent with our findings that atohlc and $1 a$ expression is reduced in arll3b-deficient embryos (Figs. 2 and S3). Moreover, wnt1 is also expressed at the midbrain-hindbrain boundary and it has been speculated to contribute to the development of the cerebellum. Whether the boundary Wnt1 also contributes to the cerebellar defects in arll3b-deficient embryos needs further experiments. Lithium treatment partially rescues the cerebellar defects in both zebrafish arl13b (Fig. 6) and mouse Ahil mutants [2], suggesting that Wnt signaling is a conserved key pathway regulating early cerebellar development and could serve as a common potential therapy target in JS. Atoh1 and Wnt signaling might only partially contribute to the mechanisms of Arl13b signaling since Arl13b has been found to be distributed subcellularly in the cilium and cytoplasm and is expressed in many tissues and cells.

Most JS research has focused on granule cells and has seldom dealt with Purkinje cells. In human JS samples, the migration of cerebellar Purkinje cells is faulty, with heterotopic and locally-interrupted Purkinje cell layers [42]. The malformation of the Purkinje cell monolayer has also been found in JS gene mutant mice [3, 43]. These late Purkinje cell defects could be due to an early developmental deficiency. It has been demonstrated that mutations of the JS gene Zfp423/ZNF423 in the mouse impair the early development of Purkinje cell progenitors [44]. Our results also demonstrated that the progenitors of Purkinje cells were selectively disrupted in the dorsomedial cluster in $a r l 13 b$-defecient embryos (Fig. 4), like the early defects in granule cell progenitors. The coincident selective defects of Purkinje and granule cell progenitors suggest that these two populations interact or share common mechanisms. Radial glia and the Bergmann glial cells derived from them provide a scaffold for the migration of both Purkinje cells 
and granule cells early in cerebellar development [45, 46]. Ciliary Arl13b has been shown to be critical for the polarization of the radial glial scaffold and the laminar organization of neurons in mouse cerebral cortex [6]. The available evidence and our current results suggest that the role of Arl13b in neural development is conserved between species and Arl13b participates in the polarization of the radial glial scaffold as well as coordinating the proliferation and migration of both Purkinje cells and granule cells early in cerebellar development. The migration of Purkinje cells and granule cells also shares molecular mechanisms, such as Reelin signaling [45], the expression level of which was also selectively reduced in the dorsomedial cerebellum in arl13b-deficient embryos (Fig. 3).

\section{Conclusions}

We have established a JS model using arll3b-deficient zebrafish, which recapitulate some of the signs of JS, such as locomotor and cerebellar developmental defects. The disruption of $\operatorname{arll} 3 \mathrm{~b}$ resulted in a dramatic reduction of granule cells specifically in the $\mathrm{CCe}$, a structure homologous to the human cerebellar vermis. The expression of the proneural gene atohl in a subpopulation of granule cells was down-regulated in the cerebellum. The early development of Purkinje cells was also selectively disrupted in the dorsomedial cerebellum. wnt 1 , a gene transiently expressed early in cerebellar development, was dramatically downregulated. Furthermore, activating Wnt signaling mitigated the granule cell defects caused by arll3b-disruption. Our results reveal the critical role of $\operatorname{arll} 13 \mathrm{~b}$ in the early development of cerebellar granule and Purkinje cells. We propose that the arll3b-deficient zebrafish can serve as a powerful tool to investigate the pathological mechanisms underlying JS.

Acknowledgements This work was supported by grants from the National Natural Science Foundation of China (31171044, 81160144, and 81760216) and the Young Scientist Project of Jiangxi Province, China (20122BCB23007). We thank Dr. Zhao-Xia Sun (Yale University) for kindly providing the arl13b mutants, Dr. Jing-Wei Xiong (Peking University) for the $T g$ (neurodl:EGFP) transgenic fish, and Dr. Sheng-Ping L. Hwang (Institute of Cellular and Organismic Biology, Academia Sinica, Taipei, China) for kindly providing plasmids. We specially thank Professor Shi-Wen Luo (Nanchang University) for critical discussion and valuable advice.

Conflict of interest The authors declare that they have no conflicts of interest.

Open Access This article is licensed under a Creative Commons Attribution 4.0 International License, which permits use, sharing, adaptation, distribution and reproduction in any medium or format, as long as you give appropriate credit to the original author(s) and the source, provide a link to the Creative Commons licence, and indicate if changes were made. The images or other third party material in this article are included in the article's Creative Commons licence, unless indicated otherwise in a credit line to the material. If material is not included in the article's Creative Commons licence and your intended use is not permitted by statutory regulation or exceeds the permitted use, you will need to obtain permission directly from the copyright holder. To view a copy of this licence, visit http://creativecommons. org/licenses/by/4.0/.

\section{References}

1. Romani M, Micalizzi A, Valente EM. Joubert syndrome: congenital cerebellar ataxia with the molar tooth. Lancet Neurol 2013, 12: 894-905.

2. Lancaster MA, Gopal DJ, Kim J, Saleem SN, Silhavy JL, Louie CM, et al. Defective Wnt-dependent cerebellar midline fusion in a mouse model of Joubert syndrome. Nat Med 2011, 17: 726-731.

3. Bashford AL, Subramanian V. Mice with a conditional deletion of Talpid3 (KIAA0586) — a model for Joubert syndrome. J Pathol 2019, 248: 396-408.

4. Cantagrel V, Silhavy JL, Bielas SL, Swistun D, Marsh SE, Bertrand JY, et al. Mutations in the cilia gene ARL13B lead to the classical form of Joubert syndrome. Am J Hum Genet 2008, 83: $170-179$.

5. Higginbotham H, Eom TY, Mariani LE, Bachleda A, Hirt J, Gukassyan V, et al. Arl13b in primary cilia regulates the migration and placement of interneurons in the developing cerebral cortex. Dev Cell 2012, 23: 925-938.

6. Higginbotham H, Guo J, Yokota Y, Umberger NL, Su CY, Li J, et al. Arl13b-regulated cilia activities are essential for polarized radial glial scaffold formation. Nat Neurosci 2013, 16: 1000-1007.

7. Caspary T, Larkins CE, Anderson KV. The graded response to sonic Hedgehog depends on cilia architecture. Dev Cell 2007, 12: 767-778.

8. Song P, Dudinsky L, Fogerty J, Gaivin R, Perkins BD. Arl13b interacts with Vangl2 to regulate cilia and photoreceptor outer segment length in zebrafish. Invest Ophthalmol Vis Sci 2016, 57 : $4517-4526$.

9. Duldulao NA, Lee S, Sun Z. Cilia localization is essential for in vivo functions of the Joubert syndrome protein Arl13b/ Scorpion. Development 2009, 136: 4033-4042.

10. Zhu L, Chen L, Yan L, Perkins BD, Li S, Li B, et al. Mutant Ahi1 affects retinal axon projection in zebrafish via toxic gain of function. Front Cell Neurosci 2019, 13: 81.

11. Kimmel CB, Ballard WW, Kimmel SR, Ullmann B, Schilling TF. Stages of embryonic development of the zebrafish. Dev Dyn 1995, 203: 253-310.

12. Liu ZZ, Zhu J, Wang CL, Wang X, Han YY, Liu LY, et al. CRMP2 and CRMP4 are differentially required for axon guidance and growth in zebrafish retinal neurons. Neural Plast 2018, 2018: 8791304.

13. Mu Z, Zhang S, He C, Hou H, Liu D, Hu N, et al. Expression of SoxC transcription factors during zebrafish retinal and optic nerve regeneration. Neurosci Bull 2017, 33: 53-61.

14. Sun Z, Amsterdam A, Pazour GJ, Cole DG, Miller MS, Hopkins $\mathrm{N}$. A genetic screen in zebrafish identifies cilia genes as a principal cause of cystic kidney. Development 2004, 131: 4085-4093.

15. Riley BB, Zhu C, Janetopoulos C, Aufderheide KJ. A critical period of ear development controlled by distinct populations of ciliated cells in the zebrafish. Dev Biol 1997, 191: 191-201. 
16. Hibi M, Shimizu T. Development of the cerebellum and cerebellar neural circuits. Dev Neurobiol 2012, 72: 282-301.

17. Kidwell CU, Su CY, Hibi M, Moens CB. Multiple zebrafish atoh1 genes specify a diversity of neuronal types in the zebrafish cerebellum. Dev Biol 2018, 438: 44-56.

18. Kani S, Bae YK, Shimizu T, Tanabe K, Satou C, Parsons MJ, et al. Proneural gene-linked neurogenesis in zebrafish cerebellum. Dev Biol 2010, 343: 1-17.

19. Volkmann K, Rieger S, Babaryka A, Koster RW. The zebrafish cerebellar rhombic lip is spatially patterned in producing granule cell populations of different functional compartments. Dev Biol 2008, 313: 167-180.

20. Aruga J, Minowa O, Yaginuma $\mathrm{H}$, Kuno J, Nagai T, Noda T, et al. Mouse Zic1 is involved in cerebellar development. J Neurosci 1998, 18: 284-293.

21. Costagli A, Kapsimali M, Wilson SW, Mione M. Conserved and divergent patterns of Reelin expression in the zebrafish central nervous system. J Comp Neurol 2002, 450: 73-93.

22. Miyata T, Maeda T, Lee JE. NeuroD is required for differentiation of the granule cells in the cerebellum and hippocampus. Genes Dev 1999, 13: 1647-1652.

23. Gao $\mathrm{H}, \mathrm{Bu} \mathrm{Y,} \mathrm{Wu} \mathrm{Q,} \mathrm{Wang} \mathrm{X,} \mathrm{Chang} \mathrm{N,} \mathrm{Lei} \mathrm{L,} \mathrm{et} \mathrm{al.} \mathrm{Mecp2}$ regulates neural cell differentiation by suppressing the Id1 to Her2 axis in zebrafish. J Cell Sci 2015, 128: 2340-2350.

24. Katsuyama Y, Oomiya Y, Dekimoto H, Motooka E, Takano A, Kikkawa S, et al. Expression of zebrafish ROR alpha gene in cerebellar-like structures. Dev Dyn 2007, 236: 2694-2701.

25. Hamling KR, Tobias ZJ, Weissman TA. Mapping the development of cerebellar Purkinje cells in zebrafish. Dev Neurobiol 2015, 75: 1174-1188.

26. Horner VL, Caspary T. Disrupted dorsal neural tube BMP signaling in the cilia mutant Arl13b hnn stems from abnormal Shh signaling. Dev Biol 2011, 355: 43-54.

27. Shao J, Xu L, Chen L, Lu Q, Xie X, Shi W, et al. Arl13b promotes gastric tumorigenesis by regulating smo trafficking and activation of the Hedgehog signaling pathway. Cancer Res 2017, 77: 4000-4013.

28. Hibi M, Matsuda K, Takeuchi M, Shimizu T, Murakami Y. Evolutionary mechanisms that generate morphology and neuralcircuit diversity of the cerebellum. Dev Growth Differ 2017, 59: 228-243.

29. McFarland KA, Topczewska JM, Weidinger G, Dorsky RI, Appel B. Hh and Wnt signaling regulate formation of olig2+ neurons in the zebrafish cerebellum. Dev Biol 2008, 318: 162-171.

30. Aldinger KA, Mendelsohn NJ, Chung BH, Zhang W, Cohn DH, Fernandez B, et al. Variable brain phenotype primarily affects the brainstem and cerebellum in patients with osteogenesis imperfecta caused by recessive WNT1 mutations. J Med Genet 2016, 53: 427-430.

31. Hagan N, Zervas M. Wnt1 expression temporally allocates upper rhombic lip progenitors and defines their terminal cell fate in the cerebellum. Mol Cell Neurosci 2012, 49: 217-229.

32. Quarello E. Enlarged intracranial translucency and molar tooth sign in the first trimester as features of Joubert syndrome and related disorders. Ultrasound Obstet Gynecol 2016, 48: 532-534.
33. Song P, Perkins BD. Developmental expression of the zebrafish Arf-like small GTPase paralogs arl13a and arl13b. Gene Expr Patterns 2018, 29: 82-87.

34. Spassky N, Han YG, Aguilar A, Strehl L, Besse L, Laclef C, et al. Primary cilia are required for cerebellar development and Shhdependent expansion of progenitor pool. Dev Biol 2008, 317: 246-259.

35. Chizhikov VV, Davenport J, Zhang Q, Shih EK, Cabello OA, Fuchs JL, et al. Cilia proteins control cerebellar morphogenesis by promoting expansion of the granule progenitor pool. J Neurosci 2007, 27: 9780-9789.

36. Ben-Arie N, Bellen HJ, Armstrong DL, McCall AE, Gordadze $\mathrm{PR}$, Guo Q, et al. Math1 is essential for genesis of cerebellar granule neurons. Nature 1997, 390: 169-172.

37. Duncan RN, Panahi S, Piotrowski T, Dorsky RI. Identification of Wnt genes expressed in neural progenitor zones during zebrafish brain development. PLoS One 2015, 10: e0145810.

38. Lucas FR, Salinas PC. WNT-7a induces axonal remodeling and increases synapsin I levels in cerebellar neurons. Dev Biol 1997, 192: 31-44.

39. Salinas PC, Fletcher C, Copeland NG, Jenkins NA, Nusse R. Maintenance of Wnt-3 expression in Purkinje cells of the mouse cerebellum depends on interactions with granule cells. Development 1994, 120: 1277-1286.

40. Shi F, Cheng YF, Wang XL, Edge AS. Beta-catenin up-regulates Atoh1 expression in neural progenitor cells by interaction with an Atoh1 3' enhancer. J Biol Chem 2010, 285: 392-400.

41. Tsuchiya K, Nakamura T, Okamoto R, Kanai T, Watanabe M. Reciprocal targeting of Hath1 and beta-catenin by Wnt glycogen synthase kinase 3 beta in human colon cancer. Gastroenterology 2007, 132: 208-220.

42. Aguilar A, Meunier A, Strehl L, Martinovic J, Bonniere M, AttieBitach T, et al. Analysis of human samples reveals impaired SHH-dependent cerebellar development in Joubert syndrome/ Meckel syndrome. Proc Natl Acad Sci U S A 2012, 109: 16951-16956.

43. Abdelhamed ZA, Abdelmottaleb DI, El-Asrag ME, Natarajan S, Wheway G, Inglehearn CF, et al. The ciliary Frizzled-like receptor Tmem67 regulates canonical Wnt/beta-catenin signalling in the developing cerebellum via Hoxb5. Sci Rep 2019, 9: 5446.

44. Casoni F, Croci L, Bosone C, D'Ambrosio R, Badaloni A, Gaudesi D, et al. Zfp423/ZNF423 regulates cell cycle progression, the mode of cell division and the DNA-damage response in Purkinje neuron progenitors. Development 2017, 144: 3686-3697.

45. Schilling K. Moving into shape: cell migration during the development and histogenesis of the cerebellum. Histochem Cell Biol 2018, 150: 13-36.

46. Xu H, Yang Y, Tang X, Zhao M, Liang F, Xu P, et al. Bergmann glia function in granule cell migration during cerebellum development. Mol Neurobiol 2013, 47: 833-844. 